\title{
Correction to: Coalescence-based species delimitation using genome-wide data reveals hidden diversity in a cosmopolitan group of lichens
}

Tetiana Lutsak $^{1}\left(\mathbb{D} \cdot\right.$ Fernando Fernández-Mendoza $^{2} \cdot$ Paul Kirika $^{3} \cdot$ Melaku Wondafrash $^{4} \cdot$ Christian Printzen $^{1}$

Published online: 24 April 2020

(C) Gesellschaft für Biologische Systematik 2020

\section{Correction to: Organisms Diversity \& Evolution} https://doi.org/10.1007/s13127-019-00424-0

The publisher regrets that the Table 3 of the original published version of this article is incorrect thus the table is now presented correctly.

The original article has been corrected.

The online version of the original article can be found at https://doi.org/ 10.1007/s13127-019-00424-0

Tetiana Lutsak

luts.ok@gmail.com

1 Senckenberg Research Institute and Natural History Museum, 60325 Frankfurt am Main, Germany

2 Institute of Plant Sciences, Karl-Franzens-Universität Graz, A-8010 Graz, Austria

3 Botany Department, National Museums of Kenya, Nairobi, Kenya

4 Biology Department, Faculty of Sciences, Addis Ababa University, Addis Ababa, Ethiopia 
Table 3 Species assignment estimations from the two runs of STACEYand tr2-delimitation. For STACEY, only the best five hypotheses are presented. Species level units are color-coded in shades of gray. * and + denote additional OTUs distinguished as separate species in suboptimal models

\begin{tabular}{|c|c|c|c|c|c|c|c|c|c|c|c|c|c|c|c|c|c|c|c|c|c|c|c|c|c|c|c|c|c|c|c|c|c|c|c|c|}
\hline \multirow{3}{*}{ 䓂 } & & \multicolumn{35}{|c|}{ Specimens and prior assignment to species } \\
\hline & \multirow{2}{*}{. } & \multirow{2}{*}{ 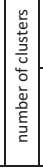 } & \multirow{2}{*}{ 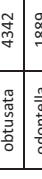 } & \multirow{2}{*}{ 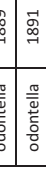 } & \multirow{2}{*}{ 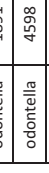 } & \multirow{2}{*}{ 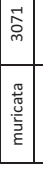 } & \multirow{2}{*}{ 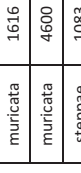 } & \multirow{2}{*}{ 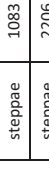 } & \multirow{2}{*}{\multicolumn{2}{|c|}{ 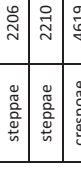 }} & ఫ̊ & \begin{tabular}{l|l}
$\overrightarrow{\tilde{g}}$ & 岁 \\
a
\end{tabular} & 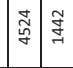 & 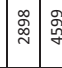 & \begin{tabular}{l|l}
$\stackrel{8}{o}$ \\
$\forall$
\end{tabular} & $\underset{\sim}{\vec{D}}$ & $\stackrel{\substack{\infty \\
\sim}}{\sim}$ & ర్ & $\vec{\nabla}$ & 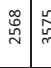 & 黛 & & 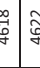 & $\begin{array}{l}y \\
\dot{y} \\
\tilde{g}\end{array}$ & 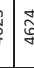 & 品 & 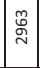 & 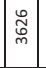 & 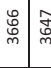 & 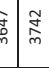 & 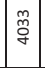 & 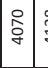 & 㗭 & $\stackrel{\circ}{\text { ฟ }}$ & $\underset{\mathscr{J}}{\widetilde{J}}$ & ปั \\
\hline & & & & & & & & & & & 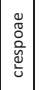 & 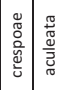 & 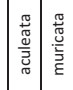 & 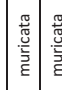 & 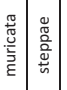 & \begin{tabular}{|l|}
$\frac{2}{0}$ \\
$\frac{0}{2}$ \\
$\frac{2}{n}$ \\
\end{tabular} & $\begin{array}{l}0 \\
\frac{0}{0} \\
\frac{2}{2} \\
\dot{5}\end{array}$ & 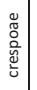 & 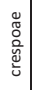 & & 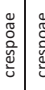 & & & & & & & 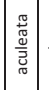 & & 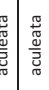 & 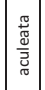 & 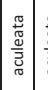 & & & 响 & \\
\hline & & & & & & & & & & & & & loci, not & accoun & unting for & or link & kage & & & & & & & & & & & & & & & & & & & \\
\hline & & & & & & & & & & & & & & STACE & & & & & & & & & & & & & & & & & & & & & & \\
\hline 15295 & 0.6126 & 6 & & & & & & & & & & & & & & & & & & & & 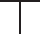 & 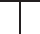 & & & & & & & & & & & & & \\
\hline 1887 & 0.0755 & 7 & & & & & & & & & & & & & & & & & & & & & & & & & & & & & & & & & * & \\
\hline 1605 & 0.0642 & 7 & & & & & & & & & & & & & * & & & * & * & * * * & *** * & * $\quad *$ & * & * & * & & & & & & & & & & & * \\
\hline 1241 & 0.0497 & 5 & & & & & & & & & & & & & & & & & & & & & & & & & & & & & & & & & & \\
\hline 483 & 0.0193 & 7 & & & & & & & * & & & & & & & & & & & & & & & & & & & & & & & & & & & \\
\hline & & & & & & & & & & & & & & r2-delimit & mitation & & & & & & & & & & & & & & & & & & & & & \\
\hline NA & NA & 6 & & & & & & & & & & & & 1 & \begin{tabular}{l|l}
1 & $\mid$ \\
\end{tabular} & & & & & & & & & & & & & & & & & & & & & \\
\hline & & & & & & & & & & & & & 24 loci, ac & ccountin & ting for $\mathrm{li}$ & linkag & & & & & & & & & & & & & & & & & & & & \\
\hline & & & & & & & & & & & & & & STACE & CEY & & & & & & & & & & & & & & & & & & & & & \\
\hline 25794 & 0.7344 & 6 & & & & & & & & & & & & & & & & & & & & & & & & & & & & & & & & & & \\
\hline 1870 & 0.0532 & 7 & & & & & & & * & & & & & & & & & & & & & & & & & & & & & & & & & & & \\
\hline 747 & 0.0213 & 7 & & & & & & & & & & & & & * & & & * & * & * & ** * & * & * & * & * & & & & & & & & & & & * \\
\hline 700 & 0.0199 & 7 & & & & & * & & & & & & & & & & & & & & & 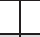 & 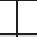 & & & & & & & & & & & & & \\
\hline 394 & 0.0112 & 7 & & & & & & & & & & & & & \begin{tabular}{|l|}
$*$ \\
\end{tabular} & \begin{tabular}{|l|l|}
$*$ \\
\end{tabular} & \begin{tabular}{l|l|}
$*$ & \\
\end{tabular} & ${ }^{*}$ & * & \begin{tabular}{l|l}
$*$ & $*$ \\
\end{tabular} & \begin{tabular}{l|l}
$*$ & $*$ \\
\end{tabular} & \begin{tabular}{l|l}
$*$ & $*$ \\
\end{tabular} & ${ }^{*}+$ & * & * & & & & & * & & & & & & * \\
\hline & & & & & & & & & & & & & & 2-delimite: & itation & & & & & & & & & & & & & & & & & & & & & \\
\hline $\mathrm{NA}$ & NA & 5 & & & & & & & & & & & & & & & & & & & & & & & & & & & & & & & & & & \\
\hline & & & & & & & & & & $20 \mathrm{lo}$ & oci, ex & xcluding & g loci unde & der poss & sssible en & nviron & nner & & selec & ction & & & & & & & & & & & & & & & & \\
\hline & & & & & & & & & & & & & & STACE & CEY & & & & & & & & & & & & & & & & & & & & & \\
\hline 1725 & 0.0453 & 6 & & & & & & & & & & & & & & & & & & & & & & & & & & & & & & & & & & \\
\hline 1429 & 0.0375 & 8 & & & & & & & $*$ & & & & & & & & & & & & & & & & & & & & & & & & & + & + & \\
\hline 820 & 0.0215 & 7 & & & & & & & & & & & & & & & & & & & & & & & & & & & & & & & & + & + & \\
\hline 355 & 0.0093 & 9 & & & & & & & * & & & & & & + & & & + & + & ++ & ++ & ++ & + & + & + & & & & & & & & & & * & + \\
\hline 254 & 0.0067 & 7 & & & & & & & * & & & & & & & & & & & & & & & & & & & & & & & & & & & \\
\hline & & & & & & & & & & & & & & r2-delimit & nitation & & & & & & & & & & & & & & & & & & & & & \\
\hline NA & NA & & & & & & & & & & & & & & & & & & & & & & & & & & & & & & & & & & & \\
\hline
\end{tabular}

